\title{
Modelling magnetically dominated and radiatively cooling jets
}

\author{
Martín Huarte-Espinosa ${ }^{1,2}$, Adam Frank $^{1}$ and Eric Blackman ${ }^{1}$ \\ ${ }^{1}$ Department of Physics and Astronomy, University of Rochester, \\ 600 Wilson Boulevard, Rochester, NY, 14627-0171 \\ emails: martinhe; afrank; blackman @pas.rochester.edu \\ ${ }^{2}$ Kavli Institute for Cosmology Cambridge, Madingley Road, \\ Cambridge CB3 0HA, UK
}

\begin{abstract}
Using 3D-MHD Eulerian-grid numerical simulations, we study the formation and evolution of rising magnetic towers propagating into an ambient medium. The towers are generated from a localized injection of pure magnetic energy. No rotation is imposed on the plasma. We compare the evolution of a radiatively cooling tower with an adiabatic one, and find that both bend due to pinch instabilities. Collimation is stronger in the radiative cooling case; the adiabatic tower tends to expand radially. Structural similarities are found between these towers and the millimeter scale magnetic towers produced in laboratory experiments.
\end{abstract}

Keywords. stars: winds, outflows, ISM: jets and outflows, methods: numerical, MHD

\section{Introduction}

Non-relativistic jets are observed in the vicinities of several Protostellar Objects, Young Stellar Objects (YSOs) and post-AGB stars. Plausible models suggest that jets are launched and collimated by accretion, rotation and magnetic mechanisms in the "central engine" (see Pudritz et al. 2007, for a review). The relative extent over which magnetic energy dominates the outflow kinetic energy in a propagating jet has traditionally divided them into two classes: magnetocentrifugal (Blandford \& Payne 1982; Ouyed \& Pudritz 1997; Blackman et al. 2001; Mohamed \& Podsiadlowski 2007), in which magnetic fields only dominate out to the Alfvén radius, or Poynting flux dominated (PFD, Lynden-Bell 1996; Ustyugova et al. 2000; Lovelace et al. 2002; Nakamura \& Meier 2004) in which magnetic fields dominate the jet structure, acting as a magnetic piston over very large distances from the engine. PFD jets carry large electric currents along which generate strong tightly wound helical magnetic fields around the jet axis. Simulations of such jets have found that these magnetic fields play a role in the formation of current-driven kink instabilities and the stabilization of Kelvin-Helmholtz $(\mathrm{KH})$ modes in jets (e.g. see Nakamura \& Meier 2004).

The effects of plasma radiative-cooling have been followed only in few simulations of magnetized, kinetic energy-dominated jets. These, have been found to form both thin cocoons and nose cones (e.g. Blondin et al. 1990; Frank et al. 1998), and to be more susceptible to KH instabilities relative to adiabatic jets (Hardee \& Stone 1997, and references therein). Correlations between the structure of kinetic energy-dominated jets and their power have been extensively explored. There has been less work on PFD jets.

Magnetic fields with initially primarily poloidal (radial and vertical) geometries anchored to accretion discs were shown to form tall, highly wound and helical magnetic structures, or magnetic towers, that expand vertically when laterally supported in pressure equilibrium with the ambient gas (Lynden-Bell 1996, 2003). The local injection of 
pure toroidal magnetic energy, without imposing any rotation on the plasma, has been shown to form magnetic towers in laboratory experiments (Lebedev et al. 2005. These experiments were modeled with numerical simulations by Ciardi et al. 2007) and are analogous to 3D-MHD numerical simulations of AGN jets by Li et al. (2006, and subsequent papers). Here we use a modified version of the implementation of $\mathrm{Li}$ et al., in order to study magnetically driven and radiatively-cooling jets, or magnetic towers, motivated by the contexts of Protostellar Objects, YSOs and Planetary Nebulae.

\section{Model}

We form magnetic towers using 3D-MHD Eulerian-grid numerical simulations by locally injecting pure magnetic energy and compare the evolution of these towers as they propagate into an ambient "interstellar" medium (ISM) for radiative cooling vs. adiabatic cases. No rotation is imposed on the plasma at the base. The ISM gas is modelled with an ideal gas equation of state, a ratio of specific heats of $\gamma=5 / 3$, a uniform number density of $100 \mathrm{~cm}^{-3}$, a constant temperature of $10000 \mathrm{~K}$ and null velocity. We start the simulations with a helical magnetic field inside a central cylinder with both radius and height of $50 \pi$ AU. The helical geometry of the initial magnetic field is described from its vector potential (i.e. $\mathbf{B}=\nabla \times \mathbf{A}$ )

$$
\mathbf{A}(r, z)=\left\{\begin{array}{cl}
\frac{r}{4}(\cos 2 r+1)(\cos 2 z+1) \hat{\phi}+\frac{\alpha}{8}(\cos 2 r+1)(\cos 2 z+1) \hat{k}, & \text { for } r, z<\pi / 2 \\
0, & \text { for } r, z \geqslant \pi / 2
\end{array}\right.
$$

in cylindrical coordinates. The parameter $\alpha$ is an integer with units of length and determines the ratio of toroidal to poloidal magnetic fluxes, and electric currents. For the initial conditions $\alpha=3$, but $\alpha=15$ at all later times.

A cubic computational domain with $128^{3}$ fixed cells is used. Boundary conditions are set to periodic at both $x= \pm 800 \mathrm{AU}$ and $y= \pm 800 \mathrm{AU}$, to reflective at $z=0$, and to outflow at $z=1600 \mathrm{AU}$. We use BlueHivet, an IBM parallel cluster of the Center for Research Computing of the University of Rochester, to run simulations for about two weeks, using 32 processors.

Using the above initial and boundary conditions, we solve the equations of radiativemagnetohydrodynamics in three-dimensions with the AMR parallel code AstroBEAR (Cunningham et al. 2009). A source term is implemented to the induction equation to continuously inject magnetic fields via (2.1), into the computational domain. For numerical stabilization, static gas is also injected into regions where $\mathbf{A}(r, z)$ is not null, but its contribution to both mass and thermal energy is negligible. The ionization of both $\mathrm{H}$ and $\mathrm{He}$, the chemistry of $\mathrm{H} 2$ and optically thin cooling are considered (in one of our simulations) using tables of Dalgarno \& McCray (1972). To focus on the minimalist physics of magnetic tower expansion, no gravitational, viscous or general-relativistic processes are considered. This implementation extends the one of Li et al. (2006) to stellar scales and to the radiative-MHD regime.

\section{Results and discussion}

Here we discuss some of our preliminary results. Higher resolution simulations, further details and analyses will be presented in Huarte Espinosa, Frank \& Blackman 2011 (in prep.).

$\dagger$ https://www.rochester.edu/its/web/wiki/crc/index.php/BlueHive_Cluster $\ddagger$ http://www.pas.rochester.edu/ bearclaw/ 


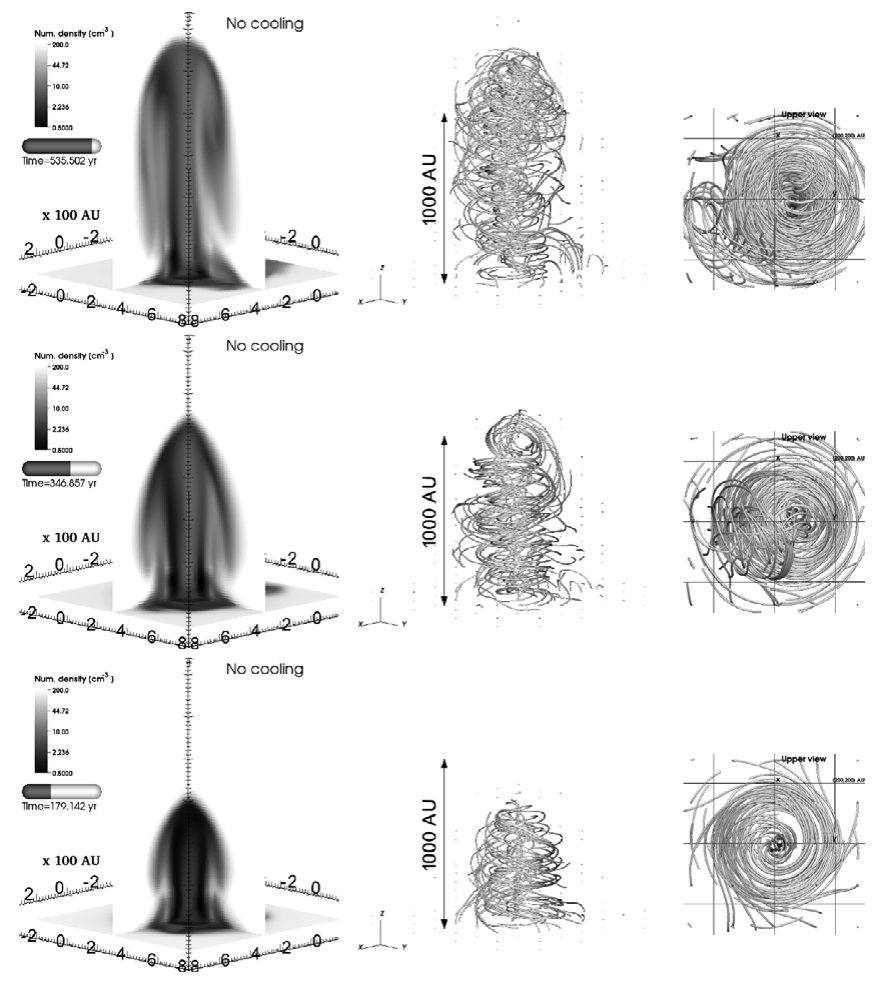

Figure 1. Evolution of the adiabatic magnetic tower. Left: logarithmic density grayscale maps. Middle: magnetic field lines. Right: field lines upper view. Time is the same row-wise. Open field lines are a visualization effect.

The adiabatic and radiatively cooling magnetic towers are shown in Figures 1 and 2, respectively. The ratio of thermal to magnetic pressures takes values within $(0.1,1)$ at regions where $r, z \leqslant 50 \pi \mathrm{AU}$, throughout the simulations. Vertical pressure gradients primarily due to the toroidal magnetic field in the tower, cause the plasma to accelerate along the $z$ direction, and a shock is driven in the plasma. A self-pinched cavity of $\sim 150 \mathrm{AU}$ is formed in about a few $10 \mathrm{yr}$ by a collimated outflow, the vertical speed of which is $\sim 100 \mathrm{~km} \mathrm{~s}^{-1}$. This "cocoon" is filled with tightly wound helical magnetic fields and gas that is about 0.01 times less dense than that of the ambient. On average, the cocoon thermal pressure is larger than the magnetic pressure by factors that increase radially from 1 to 4 , approximately, at $z \sim 300 \mathrm{AU}$. This is different than the magnetically dominated jets of $\mathrm{Li}$ et al. (2006). We note that, as opposed to other magnetized jet launch simulations (e.g. Shibata \& Uchida 1986), no rotation has been imposed on the plasma.

In the cocoon, the toroidal magnetic flux dominates the poloidal flux. A poloidal electric current develops and the radial pressure gradient from the toroidal magnetic field causes radial expansion of the adiabatic tower (see left column of Figure 1) that exceeds its collimating hoop stress. However, Figure 2 shows that cooling suppresses this effect significantly. The upper view of the magnetic field lines (rightmost column in Figures 1 and 2) shows two nested toroidal magnetic surfaces. The ambient pressure collimates the outer magnetic surface, whereas its magnetic tension collimates the field lines of the inner magnetic surface. Such structures resemble the ones seen in the millimetricscale magnetic towers produced in laboratory experiments by Ciardi et al. (2007). Pinch 


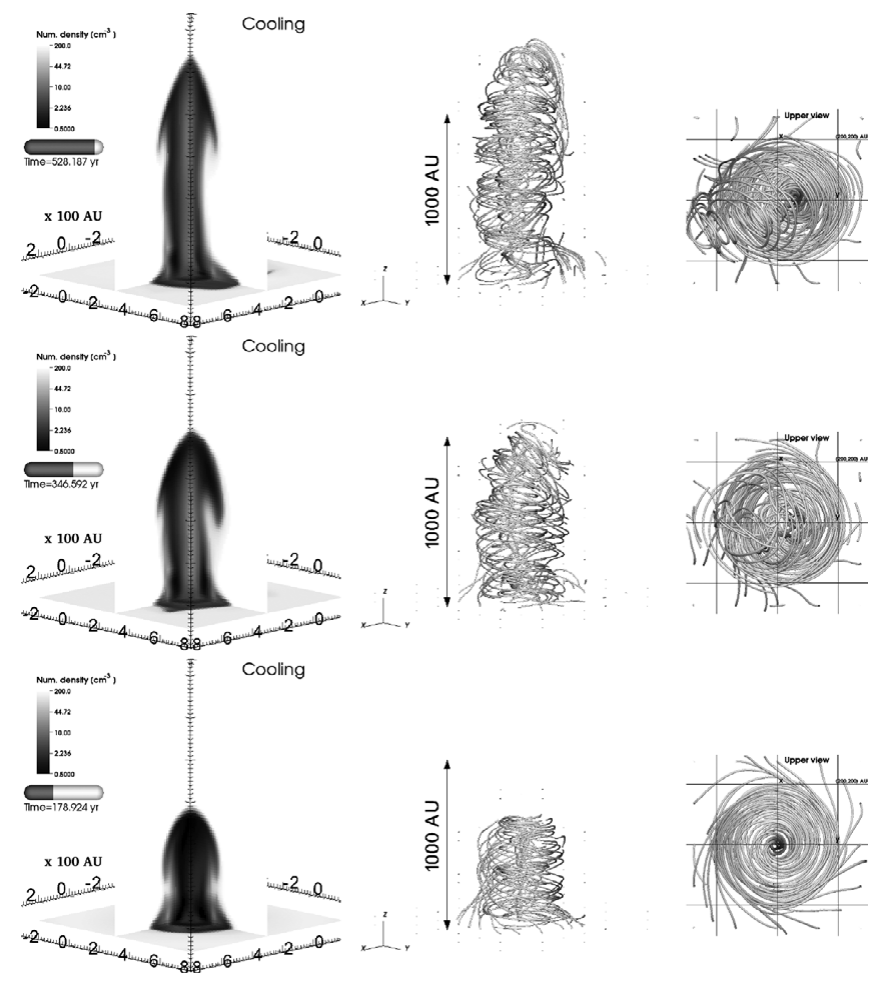

Figure 2. Evolution of the cooling magnetic tower. Panel structure is as in Figure 1.

instabilities cause the towers to bend, and poloidal field lines seem to pile up, particularly in the cooling tower.

\section{References}

Blackman, E. G., Frank, A., \& Welch, C. 2001, ApJ, 546, 288

Blandford, R. D., \& Payne, D. G. 1982, MNRAS, 199, 883

Blondin, J. M., Fryxell, B. A., \& Konigl, A. 1990, ApJ, 360, 370

Lebedev, S. V., et al. 2005, MNRAS, 361, 97

Ciardi, A., et al. 2007, Phys. of Plasmas, 14, 056501

Cunningham A. J., Frank A., Varnière P., Mitran S., \& Jones, T. W. 2009, ApJS, 182, 519

Dalgarno A. \& McCray R. A. 1972, ARA\&A, 10, 375

Frank, A., Ryu, D., Jones, T. W., \& Noriega-Crespo, A. 1998, ApJL, 494, L79

Hardee, P. E. \& Stone, J. M. 1997, ApJ, 483, 121

Li, H., Lapenta, G., Finn, J. M., Li, S., \& Colgate, S. A. 2006, ApJ, 643, 92

Lovelace, R. V. E., Li, H., Koldoba, A. V., Ustyugova, G. V., \& Romanova, M. M. 2002, ApJ, 572,445

Lynden-Bell, D. 1996, MNRAS, 279, 389

Lynden-Bell, D. 2003, MNRAS, 341, 1360

Mohamed S. \& Podsiadlowski P. 2007, ASPC, 372, 397

Nakamura, M. \& Meier, D. L. 2004, ApJ, 617, 123

Ouyed, R. \& Pudritz, R. E. 1997, ApJ, 482, 712

Pudritz, R. E., Ouyed, R., Fendt, C., \& Brandenburg, A. 2007, Protostars and Planets V, 277

Shibata, K. \& Uchida, Y. 1986, PASJ, 38, 631

Ustyugova, G. V., Lovelace, R. V. E., Romanova, M. M., Li, H., \& Colgate, S. A. 2000, ApJ, 541, L21 


\section{Discussion}

RoMERo: In the case you have as a compact object a neutron star, can you estimate the maximum field on the surace of the star necessary to allow the formation of a tower? How high is this field?

HuARTE-EsPinosA: I don't know. I suppose I could because I can control the injection of magnetic energy.

FERREIRA: YSO observations show that there is not enough pressure to confine jets. So, could magnetic towers be formed or even maintained?

Huarte-Espinosa: The fields should expand. It could be interesting to do simulations with other, weaker density profiles to see what happens to the internal magnetic surface or jet. See the comment by David Meier.

MEIER: Concerning the confinement question, manetic towers do not need large external pressure to confine them- certainly nothing like the pressure in the visible jet plasma. The main jet is confined in the foward current part of the jet. Then moving radially outward, the jet is self-confined in the current-free region. Finally, along the return current surface (from the core of the jet) the magnetic pressure is weak, and it only requires a weak ambient pressure to confine the outer magnetic tower.

YUAN: If the jet is magnetically dominated, why cooling can play an important dynamical role?

Huarte-Espinosa: Cooling can play an important role in the shock region at large distances from the central magnetized region.

FENDT: I expect reconnection being much more important than cooling as the whole field structure will be reconfigured. What do you think?

HuARTE-Espinosa: Reconnection plays a role in reality indeed. It would be interesting to redo the simulations with magnetic resistivity to see this. I honestly don't know how important reconnection would be relative to cooling. Yet, the towers in my simulations do not expand due to totation. So, line reconfiguration would not be as important as it would for a rotation-formed tower. 